\title{
Neuraxial anesthesia and multiple sclerosis
}

\author{
Anahi Perlas MD FRCPC, Vincent W. S. Chan MD FRCPC
}

$\mathrm{M}$

ULTIPLE sclerosis (MS), a demyelinating disease of the central nervous system, is characterized by episodic neurologic symptoms, often followed by fixed neurologic deficits and increasing disability over time. ${ }^{1}$ Various lines of research support the hypothesis that MS is an autoimmune disease occurring in genetically susceptible persons after an environmental, possibly viral, exposure..$^{2,3}$ Typically, this condition is characterized by focal areas of inflammation and demyelination. ${ }^{1}$ It has been postulated that activated autoreactive $\mathrm{T}$ cells traverse the blood-brain barrier, induce an inflammatory process resulting in activation of microglia and macrophages, and in turn, produce structural myelin damage and disrupt the number and distribution of $\mathrm{Na}+$ ion channels, ultimately blocking normal nerve conduction. ${ }^{4}$

Moreover, in recent years, a number of endogenous oligopeptides with sodium-channel-blocking activity have been isolated from the cerebrospinal fluid of MS patients, in concentrations that are several hundred fold greater than in normal individuals. ${ }^{5}$ Interestingly, these oligopeptides exert their blocking action by shifting the steady-state inactivation curve of the sodium channels to more negative potentials, as most local anesthetics do. ${ }^{6}$ A partial conduction blockade in the demyelinated areas is responsible for the "negative" symptoms associated with MS, such as weakness and hypoesthesia. Ectopic impulses generated at the site of demyelination are considered responsible for "positive" symptoms experienced by MS patients such as painful tonic seizures, dysesthesias, spasticity and trigeminal neuralgia. ${ }^{7}$

The pathophysiology of this condition raises important clinical questions regarding the practice of neuraxial anesthesia in these patients. First, what are the effects of local anesthetics on demyelinated fibres? Unfortunately, no studies to date have adequately addressed this question. Indirect evidence suggests that systemically administered local anesthetics may unmask silent demyelinated plaques. For example, intravenously administered lidocaine has been shown to transiently aggravate negative symptoms of the condition, allegedly by increasing the degree of conduction blockade already present in the demyelinated segments. ${ }^{8}$ Sakurai et al. administered iv lidocaine to $28 \mathrm{MS}$ patients and 19 normal subjects, reaching a mean plasma concentration of $2.7 \mu \mathrm{g} \cdot \mathrm{mL}^{-1}$. While none of the normal subjects developed symptoms, 23 of the 28 MS patients developed transient, reversible neurological symptoms, mostly visual deficits $(15 / 23)$. The presumed mechanism is that the partial sodium channel blockade achieved by this low concentration of local anesthetic is not enough to block conduction in normal central nervous system (CNS) tissue, but can exacerbate the preexisting partial blockade at the level of the de-myelinated plaque, therefore causing transient deficits to appear. It has even been suggested that the administration of systemic local anesthetics might be useful as an early diagnostic tool in the work-up of suspected MS patients. ${ }^{8}$

Moreover, systemically administered local anesthetics can alleviate the positive symptoms associated with demyelination, such as paresthesias, and spasticity, allegedly by blocking the propagation of ectopic impulses. ${ }^{7}$ Both iv lidocaine and oral mexiletine have been used successfully to treat these symptoms in patients with MS. ${ }^{7}$

The administration of intrathecal local anesthetics for the purpose of spinal anesthesia has also been reported to unmask previously silent demyelinated plaques, by precipitating the appearance of negative MS symptoms. ${ }^{9}$ Levesque reported a case of acute onset of previously undiagnosed MS presenting as oculomotor paralysis following spinal anesthesia performed for a minor orthopedic procedure. ${ }^{9}$ Although the underlying mechanism for this phenomenon is not known, it seems plausible that a low concentration of

From the Department of Anesthesia, Toronto Western Hospital, University Health Network, University of Toronto, Toronto, Ontario, Canada.

Address correspondence to: Dr. Vincent Chan, Department of Anesthesia, Toronto Western Hospital, 399 Bathurst Street, Toronto, Ontario M5T 2S8, Canada. Phone: 416-603-5118; Fax: 416-603-6494; E-mail: Vincent.Chan@uhn.on.ca 
local anesthetic reaching higher CNS structures could have unmasked a silent demyelinated plaque. These symptoms, whether unmasked by systemically or intrathecally administered local anesthetics, are typically transient and reversible, and do not imply worsening of the underlying condition, unlike a true disease relapse or exacerbation, ultimately defined by appearance of new demyelinated areas. ${ }^{7}$

Second, is the quality of neuraxial blockade any different in MS patients? Should we expect a greater intensity or duration of blockade in MS patients? To date, no animal or laboratory study has adequately addressed this question. Clinical literature on this subject consists primarily of case reports and small case series. For example, Berger reported the safe use of spinal tetracaine (10 $\mathrm{mg})$ and morphine $(500 \mu \mathrm{g})$ in a patient with MS who experienced no prolongation of block (duration less than three hours). ${ }^{10}$ Other case series did not describe the quality or duration of anesthesia. ${ }^{11,12}$

In the current issue of the Canadian Journal of Anesthesia, Finucane et al. report an unusual case of presumed unintentional spinal anesthetic following a two-level lumbar paravertebral block in a 33-yr-old lady who was deemed healthy at the time of surgery but was later found to have MS. ${ }^{13}$ Extensive sensory and motor blockade from T4 to sacrum bilaterally following the administration of bupivacaine $60 \mathrm{mg}$, made the authors suspicious of unintentional intrathecal administration. Block regression was prolonged; the level regressed to T12 six and a half hours later, and completely resolved sometime between six and a half and $11 \mathrm{hr}$ post-injection. The authors suggest that block duration was far longer than expected, although the spinal dose could have been as high as bupivacaine $60 \mathrm{mg}$. Because the patient was subsequently diagnosed with MS, the authors speculated that the prolonged block was likely related to abnormal local anesthetic uptake into the spinal cord in the presence of demyelination. In our opinion, it is difficult to draw firm conclusions from this case report since the location of local anesthetic injection is presumed, the dose was unknown, and the duration of the block was estimated.

Third, does neuraxial blockade precipitate disease relapse and have an adverse impact upon the natural course of the disease? Although controversial, the limited available data suggest that the rate of disease relapse in the postoperative or postpartum periods is not affected by the choice of anesthetic technique.

It is well known that stressful periods after surgery and childbirth are associated with high rates of relapse. ${ }^{14}$ This phenomenon is also present in other immunologic disorders such as rheumatoid arthritis and systemic lupus erythematosus. ${ }^{14}$ For example, the MS relapse rate was cited at $34 \%$ in the post-partum period. ${ }^{14}$ In a prospective series of 32 obstetric patients with a firm diagnosis of MS, Bader et al. reported the use of 14 epidurals, five general anesthetics and 13 local anesthetics for peri-partum management. The rate of relapse within three months was comparable in the three groups, and was similar to the one previously reported for women with MS in the first few months post-partum. ${ }^{11}$ Also, the scattered timing of those relapses made the author suggest that factors other than the anesthetic technique should be considered. More recently, from a series of 19 obstetric patients, Dalmas et al. suggest that epidural anesthesia is innocuous in this context. ${ }^{15}$ Crawford et al. reported 50 non-obstetric and seven obstetric lumbar epidural anesthetics with only one postoperative relapse. ${ }^{16}$ Another case series of 14 spinal anesthetics for urologic surgery reported only one case of transient worsening of symptoms, and this incidence was found to be similar to patients receiving general anesthesia. ${ }^{17}$

Finally, are there absolute contraindications of neuraxial blockade in MS patients? There may be some rare specific presentations of MS that may preclude performance of a neuraxial block. For example, the presentation of MS may mimic that of intracranial tumours and it has been suggested that MS patients may have a higher incidence of intracranial gliomas. ${ }^{18,19}$

However, based upon previous discussions, it seems reasonable that for most patients, a previous diagnosis of MS should not in itself, be a contraindication for either epidural or spinal anesthesia as formerly postulated. ${ }^{20}$ Rather, the choice of anesthetic technique should be based upon a careful consideration of risks and benefits for each specific patient. Considerations should include well established contraindications (such as coagulation abnormalities, concurrent systemic infection, increased intracranial pressure, etc.) and patient preference. Furthermore, medico-legal concerns for exacerbation of MS symptoms following neuraxial blockade should be addressed by actively engaging the patient in the decision-making process regarding the choice of anesthetic technique, and obtaining informed consent after a thorough discussion of the available options. 


\section{Anesthésie neuraxiale et sclérose en plaques}

La sclérose en plaques $(\mathrm{SP})$, une maladie démyélinisante du système nerveux central, est caractérisée par des symptômes neurologiques épisodiques, souvent suivis de déficits neurologiques persistants et d'invalidité progressive avec le temps. ${ }^{1}$ Diverses recherches appuient l'hypothèse que la SP soit une maladie autoimmune qui survient chez des personnes génétiquement susceptibles à la suite d'une exposition environnementale et possiblement virale., ${ }^{2,3}$ En général, elle est caractérisée par des foyers d'inflammation et de démyélinisation. ${ }^{1}$ On a supposé que des cellules $\mathrm{T}$ autoréactives activées traversent la barrière hémato-encéphalique, induisent un processus inflammatoire qui entraine l'activation de la microglie et de macrophages qui, à leur tour, produisent des dommages structurels à la myéline et perturbent le nombre et la distribution des canaux sodiques, bloquant finalement la conduction nerveuse normale. ${ }^{4}$

De plus, au cours des dernières années, un certain nombre d'oligopeptides endogènes, dont l'activité bloque les canaux sodiques, ont été isolés du liquide céphalo-rachidien de patients atteints de SP en concentrations plus élevées de plusieurs centaines de fois que chez des individus normaux. ${ }^{5}$ Ces oligopeptides agissent en déplaçant la courbe d'inactivation à l'état d'équilibre des canaux sodiques vers des potentiels plus négatifs, comme le font la plupart des anesthésiques locaux. ${ }^{6}$ Un blocage partiel de la conduction dans les aires démyélinisées est responsable des symptômes «négatifs» associés à la SP, comme la faiblesse et l'hypoesthésie. Des impulsions ectopiques générées au site de démyélinisation sont vues comme responsables de symptômes «positifs» chez les patients SP comme des convulsions toniques douloureuses, des dysesthésies, la spasticité et la névralgie faciale. ${ }^{7}$

La physiopathologie de ce désordre soulève d'importantes questions cliniques sur l'emploi de l'anesthésie neuraxiale chez ces patients. Premièrement, quels sont les effets des anesthésiques locaux sur les fibres démyélinisées ? Malheureusement, aucune étude jusqu'à maintenant n'a traité adéquatement de la question. Une preuve indirecte laisse croire que l'administration systémique d'anesthésiques locaux pourrait démasquer des plaques démyélinisées silencieuses. Ainsi, on a montré que l'administration intraveineuse de lidocaïne a provoqué l'aggravation transitoire des symptômes négatifs de la maladie, probablement en augmentant le degré de blocage de la conduction déjà présent dans les segments démyélinisés. ${ }^{8}$ Sakurai et coll. ont administré de la lidocaïne $i v$ à 28 patients $\mathrm{SP}$ et à 19 sujets normaux, pour une concentration plasmatique de $2,7 \mu \mathrm{g} \cdot \mathrm{mL}^{-1}$. Aucun sujet normal n'a eu de symptômes mais 23 des 28 patients SP ont présenté des déficits neurologiques transitoires et réversibles, surtout visuels $(15 / 23)$. Le mécanisme présumé est que le blocage partiel des canaux sodiques produit par la faible concentration d'anesthésique local n'est pas suffisant pour bloquer la conduction dans le tissu normal du système nerveux central (SNC), mais il peut exacerber le blocage partiel préexistant au niveau de la plaque démyélinisée, faisant ainsi apparaitre des déficits transitoires. On a pensé que l'administration d'anesthésiques locaux systémiques pouvait être utile comme outil de diagnostic précoce lors de l'examen de patients chez qui on soupçonne une SP. ${ }^{8}$

Qui plus est, l'administration systémique d'anesthésique local peut atténuer les symptômes positifs associés à la démyélinisation comme la paresthésie et la spasticité, probablement en bloquant la propagation d'impulsions ectopiques. ${ }^{7}$ La lidocaïne et la mexilétine iv ont été toutes deux utilisées avec succès pour traiter ces symptômes chez des patients atteints de SP. ${ }^{7}$

L'administration intrathécale d'anesthésique local pour les besoins de la rachianesthésie a aussi permis de découvrir des plaques démyélinisées silencieuses en précipitant l'apparition de symptômes négatifs de SP. ${ }^{9}$ Levesque a rapporté le cas d'un début soudain de SP non diagnostiquée antérieurement qui s'est présenté comme une paralysie oculomotrice après une rachianesthésie réalisée pour une intervention chirurgicale orthopédique mineure. ${ }^{9}$ Bien que le mécanisme à l'origine de ce phénomène ne soit pas connu, il est plausible qu'une faible concentration d'anesthésique local ait atteint des structures supérieures du SNC et démasqué une plaque démyélinisée silencieuse. Ces symptômes, démasqués par l'administration systémique ou intrathécale d'anesthésiques locaux, sont en général transitoires et réversibles et n'impliquent pas d'aggravation de l'état sous-jacent, à la différence d'une véritable récidive ou exacerbation de la maladie, finalement déterminée par l'apparition de nouvelles aires démyélinisées. ${ }^{7}$

Deuxièmement, est-ce que la qualité du blocage neuraxial est différente d'un patient SP à l'autre ? Devrions-nous nous attendre à une plus grande intensité ou durée du blocage chez les patients SP ? Jusqu'à maintenant, aucun étude chez les animaux ou en la- 
boratoire n'a vraiment abordé cette question. La documentation clinique à ce sujet est principalement constituée de présentations de cas et de petites séries. Ainsi, Berger a rapporté l'usage sûr de tétracaïne (10 $\mathrm{mg})$ et de morphine $(500 \mu \mathrm{g})$ rachidiennes chez un patient SP chez qui il n'y a pas eu de prolongation du bloc (durée de moins de trois heures). ${ }^{10}$ Dans d'autres séries on n'a pas décrit la qualité ou la durée de l'anesthésie. ${ }^{11,12}$

Dans le présent numéro du Journal canadien d'anesthésie, Finucane et coll. présentent un cas inhabituel de rachianesthésie accidentelle présumée à la suite d'un bloc paravertébral lombaire à deux niveaux chez une femme de 33 ans jugée en bonne santé au moment de l'intervention chirurgicale, mais chez qui on a diagnostiqué une SP par la suite. ${ }^{13}$ Le bloc sensitif et moteur, bilatéralement étendu de T4 au sacrum, suivant l'administration de $60 \mathrm{mg}$ de bupivacaïne a rendu les auteurs perplexes au sujet d'une possible administration intrathécale involontaire. La régression du bloc s'est prolongée ; il a régressé à $\mathrm{T} 12$, six heures et demie plus tard et a été complètement résolu de six heures et demie à onze heures après l'injection. Les auteurs pensent que la durée du bloc a été beaucoup plus longue que prévue, même si la dose rachidienne avait été aussi élevée que $60 \mathrm{mg}$ de bupivacaïne. Comme un diagnostic de SP a été posé ultérieurement, les auteurs ont supposé que la prolongation du bloc puisse être reliée à la capture anormale d'anesthésique local dans la moelle épinière en présence de démyélinisation. Il est difficile de se prononcer fermement sur cette présentation de cas, car l'aire d'injection de l'anesthésique local est présumée, la dose est inconnue et la durée du bloc estimée.

Troisièmement, le blocage neuraxial provoque-t-il une récidive et a-t-il un effet indésirable sur le cours normal de la maladie ? Bien que cela prête à controverse, les données limitées disponibles suggèrent que le taux de récidive de la maladie en périodes postopératoire et postpartum ne soit pas influencé par le choix de la technique anesthésique.

Il est bien connu que le stress qui suit une intervention chirurgicale et un accouchement est associé à des taux élevés de récidive. ${ }^{14}$ Ce phénomène est aussi présent dans d'autres désordres immunologiques comme l'arthrite rhumatoïde et le lupus érythémateux disséminé. ${ }^{14}$ Par exemple, le taux de récidive de SP a été cité à $34 \%$ pour le post-partum. ${ }^{14}$ Dans une série prospective de 32 patientes d'obstétrique atteintes de SP diagnostiquée, Bader et coll. ont rapporté 14 anesthésies péridurales, 5 anesthésies générales et 13 anesthésies locales en période périnatale. Le taux de récidive en moins de trois mois était comparable dans les trois groupes et similaire à celui qu'on avait déjà rapporté pour des femmes avec SP pendant les premiers mois du post-partum. ${ }^{11}$ Aussi, le rythme diffus de ces poussées fait dire aux auteurs qu'il faut tenir compte d'autres facteurs que la technique anesthésique. Plus récemment, partant d'une série de 19 patientes d'obstétrique, Dalmas et coll. suggèrent que l'anesthésie péridurale soit inoffensive dans ce contexte. ${ }^{15}$ Crawford et coll. ont consigné 50 anesthésies non obstétricales et 7 anesthésies lombaires obstétricales suivies d'une seule récidive postopératoire. ${ }^{16}$ Une autre série de 14 rachianesthésies réalisées en chirurgie urologique a montré un seul cas d'aggravation transitoire des symptômes et l'incidence était similaire pour des patients qui avaient eu une anesthésie générale. ${ }^{17}$

Finalement, y a-t-il des contre-indications absolues au blocage neuraxial chez des patients SP? Certaines manifestations spécifiques rares de la SP pourraient empêcher le fonctionnement d'un bloc neuraxial. Par exemple, l'expression de la SP peut être analogue à celle des tumeurs intracrâniennes, ce qui a amené à penser que les patients SP pourraient présenter une plus grande incidence de gliomes intracrâniens. ${ }^{18,19}$

Cependant, sur la base des discussions précédentes, on peut raisonnablement croire que pour la majorité des patients un diagnostic antérieur de SP ne peut en lui-même être une contre-indication à quelque anesthésie péridurale ou rachidienne que ce soit comme on l'a supposé précédemment. ${ }^{20}$ Le choix de la technique anesthésique devrait plutôt se fonder sur un examen attentif des risques et des avantages particuliers à chaque patient. Il faudrait tenir compte de contre-indications bien établies (comme les anomalies de coagulation, une infection systémique concurrente, de l'hypertension intracrânienne, etc.) et de la préférence du patient. Il faudrait aussi aborder les questions médico-légales sur l'exacerbation des symptômes de la SP après un blocage neuraxial en engageant activement le patient dans le choix de la technique anesthésique et en obtenant un consentement éclairé après une discussion approfondie des options offertes.

\section{References}

1 Rudick RA, Cohen JA, Weinstock-Guttman B, Kinkel $R P$, Ransoboff RM. Management of multiple sclerosis. N Engl J Med 1997; 337: 1604-11.

2 Sibley WA, Bamford CR, Clark K. Clinical viral infections and multiple sclerosis. Lancet 1985; 1: 1313-5.

3 Sadovnick AD, Ebers GC. Genetics of multiple sclerosis. Neurol Clin 1995; 13: 99-118.

4 Waxman SG, Cummins TR, Black JA, Dib-Hajj S. 
Diverse functions and dynamic expression of neuronal sodium channels. Novartis Found Symp 2002; 241 : 34-51; discussion 51-60.

5 Weber F, Rudel R, Aulkemeyer P, Brinkmeier $H$. The endogenous pentapeptide QYNAD induces acute conduction block in the isolated rat sciatic nerve. Neurosci Let 2002; 317: 33-6.

6 Brinkmeier H, Aulkemeyer P, Wollinsky KH, Rudel R. An endogenous pentapeptide acting as a sodium channel blocker in inflammatory autoimmune disorders of the central nervous system. Nat Med 2000; 6: 808-11.

7 Sakurai M, Kanazawa I. Positive symptoms in multiple sclerosis: their treatment with sodium channel blockers, lidocaine and mexiletine. J Neurol Sci 1999; 162: 162-8.

8 Sakurai M, Mannen T, Kanazawa I, Tanabo H. Lidocaine unmasks silent demyelinative lesions in multiple sclerosis. Neurology 1992; 42: 2088-93.

9 Levesque P, Marsepoil T, Venutolo PH, Lesouef JM. Multiple sclerosis revealed by spinal anesthesia (French). Ann Fr Anesth Reanim 1988; 7: 68-70.

10 Berger JM, Ontell R. Intrathecal morphine in conjuction with a combined spinal and general anesthetic in a patient with multiple sclerosis. Anesthesiology 1987; 66: 400-2.

11 Bader AM, Hunt CO, Datta S, Naulty S, Ostheimer $G W$. Anesthesia for the obstetric patient with multiple sclerosis. J Clin Anesth 1988; 1: 21-4.

12 Kytta J, Rosenberg PH. Anaesthesia for patients with multiple sclerosis. Ann Chir Gynaecol 1984; 73 : 299-303.

13 Finucane BT, Terblanche OC. Prolonged duration of anesthesia in a patient with multiple sclerosis following paravertebral block. Can J Anesth 2005; 52: 493-7.

14 Nelson LM, Franklin GM, Jones MC. Anesthesia and multiple sclerosis (Letter). JAMA 1988; 260: 2838.

15 Dalmas AF, Texier C, Ducloy-Bouthors AS, KrivosicHorber $R$. Obstetrical analgesia and anaesthesia in multiple sclerosis (French). Ann Fr Anesth Reanim 2003; 22: 861-4.

16 Crawford JS, James FM III, Nolte H, Van Steenberge A, Shah JL. Regional analgesia for patients with chronic neurological disease and similar conditions. Anaesthesia 1981; 36: 821 .

17 Bouchard P, Caillet JB, Monnet F, Banssillon V. Spinal anaesthesia and multiple sclerosis (French). Ann Fr Anesth Reanim 1984; 3: 194-8.

18 Di Patre PL, Castillo V, Delavelle J, Vuillemoz S, Picard $F$, Landis T. "Tumor-mimicking" multiple sclerosis. Clin Neuropathol 2003; 22: 235-9.

19 Shuangshoti S, Hjardermaal GM, Ahmad $\Upsilon$, Arden JL, Herman MM. Concurrence of multiple sclerosis and intracranial glioma. Report of a case and review of the literature. Clin Neuropathol 2003; 22: 304-8.

20 Bamford C, Sibley W, Laguna J. Anesthesia in multiple sclerosis. J Can Sci Neurol 1978; 5: 41-4. 